\title{
Correspondence
}

\section{Measurement of environmental complexity by CAMHS}

Thompson et $a l^{1}$ considered the use of the Paddington Complexity Scale ${ }^{2}$ to quantify the case complexity of their child and adolescent mental health service (CAMHS) attenders, but discarded it largely because their study was based on CAMHS records which did not record systematically information on diagnoses or other items relevant to multi-axial formulations, such as physical health and cognitive/ developmental status of children and young people seen.

I would like to clarify that - to allow for the fact that CAMHS vary in the extent to which diagnostic and other detailed comprehensive information is obtained on cases seen by different professionals - the Paddington Complexity Scale has, in addition to a summative total score, two complexity subscores and scales: clinical (in itself subdivided into psychiatric, incorporating diagnosis, severity/duration, comorbidity items, and physical/development, scoring information on physical health and intellectual disability) and environmental (with items on family status and attitudes to the use of the service, type of school and multi-agency involvement). These subscales, in particular adaptations of the environmental subscale which is made up of items that will be known to most CAMHS workers, can and have been used on a stand-alone basis, as a measure of psychosocial case complexity. ${ }^{3}$

1 Thompson AE, Nadkarni A, Nazir SA, Sorour W, Owen V, Buggineni SK. Professional life in child and adolescent mental health services may be getting more complicated, but cases are not. Psychiatrist 2013; 37 326-30.

2 Yates P, Garralda ME, Higginson I. Paddington Complexity Scale and Health of the Nation Outcome Scales for Children and Adolescents. Br J Psychiatry 1999; 174: 417-23.

3 Audit Commission. Children in Mind: Child and Adolescent Mental Health Services (National Report). Audit Commission, 1999 (http://archive.audit-commission.gov.uk/auditcommission/subwebs/ publications/studies/studyPDF/1350.pdf).

M. Elena Garralda, Emeritus Professor, Imperial College London, email: e.garralda@imperial.ac.uk

doi: $10.1192 / \mathrm{pb} .38 .2 .86$

\section{Quality assurance in mental health clustering for PbR or the 'national tariff' - a slave with many masters}

I believe the quality assurance of the clustering process (using the Mental Health Clustering Tool (MHCT) incorporating the Health of the Nation Outcome Scales (HoNOS)) is a complex field. Bekas \& Michev' have approached it from the MHCT 'red rule' perspective and the ICD-10 coding perspective. What the results show is that to comply with one you might potentially be in breach of the other. We face this in clinical practice; for example, bipolar affective disorder is considered to be a 'psychotic' condition, although as clinicians we all know there are times when patients with bipolar affective disorder are not psychotic. On such occasions, if you rate them on the MHCT they might score ' $\mathrm{O}$ ' and then if you cluster them in a psychotic cluster you breach the 'red rule' and if you do not, you breach the ICD-10 coding expectations.

There would be another layer of complexity added when all the 'care packages' are agreed between the commissioners and providers. I am sure there would be interest to ensure that the care provided or offered reflects the package agreed. The elusive 'gold standard' that the authors allude to, if developed, cannot be one-dimensional. It needs to clarify, when there are conflicting standards, that the clinician has to adhere to the one which takes priority and therefore in my opinion should be hierarchical. In fact, the authors of the MHCT might consider dropping the 'red rules' which might have outlived their usefulness when there are agreed care packages in place. Until then the MHCT and the clustering process remain imperfect tools that clinicians have to navigate to communicate with the commissioners.

Declaration of interest: R.B. is one of the clinical leads in payment by results for East London NHS Foundation Trust.

1 Bekas S, Michev O. Payment by results: validating care cluster allocation in the real world. Psychiatrist 2013; 37: 349-55.

Rahul Bhattacharya, Consultant Psychiatrist, East London NHS Foundation Trust, email: rahul.bhattacharya@nhs.net

doi: $10.1192 / p b .38 .2 .86 a$

\section{Not everything that counts can be counted and not everything that can be counted counts}

In their excellent paper, Bekas \& Michev ${ }^{1}$ present a sober assessment of the inherent weakness of the Mental Health Clustering Tool and ICD-10 coding. Although clustering has already been used for many years in acute care, what is suitable for acute care is not necessary applicable to psychiatry. We are expected to cluster patients with similar symptoms, needs and disabilities in 21 clusters which are used as the basis for financial funding.

However, subjectivity in psychiatry is a fact and it does not really matter how many tools and scales we implement to change this. The chance of subjectivity may be reduced but never eliminated. Diagnosis and formulations vary between clinicians within the same profession and even between members of the same team. One can identify quite easily a sizeable number of patients with an ever-changing diagnosis over a number of admissions. It follows that clustering is not a static tag but a changeable process that ought to be regularly updated.

I agree wholeheartedly with Bekas \& Michev that the final arbiter should be clinical judgement. It is not uncommon practice for clinicians such as myself to override the cluster concluded by other members of the team, relying on and trusting my clinical judgement.

1 Bekas S, Michev O. Payment by results: validating care cluster allocation in the real world. Psychiatrist 2013; 37: 349-55.

Salwa Khalil, Consultant Psychiatrist, Hallam Street Hospital, West Bromwich, UK, email: salwa.khalil@bcpft.nhs.uk

doi: 10.1192/pb.38.2.86b 